Conclusion The overwhelming etiology of urethritis among HIV-infected men in Malawi is Neisseria gonorrhoeae. Current syndromic management guidelines that treat gonorrhea, chlamydia and trichomonas seem adequate for treatment of UD but future guidelines must be informed by ongoing monitoring of antibiotic resistance.

Disclosure No significant relationships.

\section{P793 RISK FACTORS FOR INCIDENT NON-GONOCOCCAL URETHRITIS (NGU) IN MEN WHO HAVE SEX WITH WOMEN (MSW) ATTENDING AN STD CLINIC}

\begin{abstract}
'Emily Rowlinson*, 'Laura Chambers, ${ }^{2}$ Sylvan Lowens, ${ }^{2}$ Jennifer Morgan, ${ }^{1}$ Tashina Robinson, 'Sarah Romano, 'Gina Leipertz, ${ }^{3}$ Matthew Golden, ${ }^{4}$ James Hughes, ${ }^{5}$ Lisa Manhart. 'University of Washington, Epidemiology, Seattle, USA; ${ }^{2}$ Public Health Seattle and King County, Seattle, USA; ${ }^{3}$ University of Washington, Medicine, Seattle, USA; ${ }^{4}$ University of Washington, Biostatistics, Seattle, USA; ${ }^{5}$ University of Washington, Epidemiology, Global Health, Seattle, USA
\end{abstract}

\subsection{6/sextrans-2019-sti.847}

Background Incidence and risk factors for NGU remain poorly defined. We conducted a cohort study to estimate incidence and identify associated risk factors in MSW.

Methods We enrolled cisgender male STD clinic patients age $\geq 16$, who reported exclusively female partners. At enrollment and six monthly follow-up visits, men underwent a clinical exam, provided urethral swab and urine specimens, and completed a sexual behavior survey. We tested for chlamydia (CT) and Mycoplasma genitalium (MG) using Aptima. NGU was defined as urethral symptoms or visible discharge plus $\geq 5$ polymorphonuclear leukocytes per high-power field on a Gram-stained slide. NGU following an NGU-negative visit was considered incident. We estimated incidence of NGU overall, pathogen-associated (MG or CT) and idiopathic NGU using Poisson regression for clustered outcomes. We performed relative risk binomial regression for clustered data to identify characteristics associated with incident NGU.

Results From 08/2014-08/2018, 254 participants had $\geq 1$ follow-up visit, contributing 100.6 person-years at risk during follow-up. Median age was 32 (range=17-71), 53\% were white and 24\% black. Eighty-four (33\%) had NGU at enrollment. Forty-five men had 53 cases of incident NGU (incidence $=0.53$ per person-year $(95 \%$ confidence interval $[\mathrm{CI}]$ $=0.39-0.71)$ ). Incidence of pathogen-associated and idiopathic NGU was 0.06 (95\% CI $0.03-0.13$ ) and 0.47 (95\%CI $=0.34-0.63)$, respectively. After adjustment for age, condom use and new partners during follow-up, risk of incident NGU was higher among black men (adjusted RR $(A R R)=2.2$; $95 \% \mathrm{CI}=1.1-4.4)$, those with a history of NGU before enrollment $(\mathrm{ARR}=3.1 ; 1.5-6.5)$ and more sex partners during follow-up $(A R R=1.2$ per partner; 1.0-1.5); risk was lower among men who used lubricant at last sex $(A R R=0.44$; 0.20-0.96).

Conclusion Incidence of NGU was high, predominantly idiopathic, and associated with traditional socio-behavioral characteristics, but not age, condom use, or new partners. The lubricant-use association was unexpected and warrants further exploration. More precise daily diary data may yield additional insights.

Disclosure No significant relationships.
P794 SIGNS AND SYMPTOMS ASSOCIATED WITH SINGLEPATHOGEN NONGONOCOCCAL URETHRITIS IN MEN

${ }^{1}$ Teresa Batteiger*, ${ }^{2}$ Stephen Jordan, ${ }^{3}$ Evelyn Toh, ${ }^{2}$ James Williams, ${ }^{3}$ Lora Fortenberry, ${ }^{1}$ Byron Batteiger, ${ }^{3}$ David Nelson. ${ }^{1}$ Indiana University School of Medicine, Medicine, Division of Infectious Diseases, Indianapolis, USA; ${ }^{2}$ Indiana University School of Medicine, Infectious Diseases, Indianapolis, USA; ${ }^{3}$ Indiana University School of Medicine, Microbiology and Immunology, Indianapolis, USA

\subsection{6/sextrans-2019-sti.848}

Background Syndromic management remains the standard nongonococcal urethritis (NGU) treatment approach. Whether pathogen-specific signs/symptoms inform treatment decisions remains unclear. We identified men with single- and mixedpathogen NGU and assessed for the presence of pathogen-specific signs or symptoms to improve syndromic management.

Methods As part of an ongoing cohort study (the Idiopathic Urethritis Men's Project [IUMP]), we recruited men with NGU. NGU was diagnosed by signs and/or symptoms of urethritis, and a urethral Gram stain with $\geq 5$ neutrophils per high-power field without evidence of gram negative intracellular diplococci. Participants underwent a clinical history and physical exam, which documented specific self-reported symptoms and clinician observed signs. Single- and mixed-infections were identified by NAAT testing of first-catch urine for Neisseria gonorrhoeae (NG), Chlamydia trachomatis (CT), Mycoplasma genitalium (MG), Trichomonas vaginalis (TV), and Ureaplasma urealyticum (UU); five-pathogen-negative cases were classified as idiopathic urethritis (IU).

Results One hundred fifty-five men with NGU are included in this analysis. The median age was 28 (range 18-63), 101 (65\%) were African American, and 135 (87\%) self-identified as heterosexual. The most commonly reported symptom was urethral discharge (92\%), followed by burning/tingling (37\%), and dysuria (28\%). Over half of these men reported more than one symptom (58\%). Single-pathogen NGU was detected in $99(64 \%)$ men, mixed-pathogen in $14(9 \%)$, and IU in 42 (27\%). For single pathogen NGU, 53 (34\%) had CT, 26 (17\%) had MG, 3 (2\%) had TV, and 17 (11\%) had UU. We compared single-pathogen NGU, mixed-infection and IU for differences in signs or symptoms and found no pathogen-specific differences.

Conclusion In men with NGU, no pathogen-specific signs and symptoms were identified that could inform treatment decisions. Pathogen-specific point-of-care tests are needed.

Disclosure No significant relationships.

\section{P795 PREVALENCE AND ETIOLOGY OF POST-AZITHROMYCIN PERSISTENT NON-GONOCOCCAL URETHRITIS (NGU) SYMPTOMS IN MEN}

${ }^{1}$ Stephen Jordan*, ${ }^{2}$ Evelyn Toh, ${ }^{3}$ Teresa Batteiger, ${ }^{1} J a m e s$ Williams, 2 Lora Fortenberry, ${ }^{1}$ Byron Batteiger, ${ }^{2}$ David Nelson. ${ }^{1}$ Indiana University School of Medicine, Medicine, Division of Infectious Diseases, Indianapolis, USA; 'Indiana University School of Medicine, Microbiology and Immunology, Indianapolis, USA; ${ }^{3}$ Indiana University School of Medicine, Indianapolis, USA

\subsection{6/sextrans-2019-sti.849}

Background Persistent NGU occurs when symptoms persist after empiric NGU treatment and has been associated with Mycoplasma genitalium (MG) infection. The prevalence and etiology of persistent NGU in men remains largely unknown. 